\title{
Spin ladders with nonmagnetic impurities
}

\author{
H.-J. Mikeska, U. Neugebauer \\ Institut für Theoretische Physik, Universität Hannover, Appelstraße 2, $3016^{r}$ Hannover, Germany \\ U. Schollwöck \\ Sektion Physik, Ludwig-Maximilians-Universität München, Theresienstraße 37, 80333 München, \\ Germany
}

\begin{abstract}
We investigate antiferromagnetic spin ladders with nonmagnetic impurities by variational and numerical (Lanczos and DMRG) methods. The interaction between the two unpaired spins opposite to the impurities is described by an effective exchange interaction $J_{\text {eff }}$, the magnitude of which depends on the impurity distance. The magnitude of $J_{\text {eff }}$ is different for unpaired spins at the edges of an open ladder and in the bulk. This difference is related to the different distribution of the unpaired spin into the bulk of the ladder. The numerical results are interpreted using matrix product states. Using the DMRG we calculate the spectrum of low-lying energy levels for up to 6 impurities and find that these spectra can be reproduced assuming pair interactions with an accuracy of better than $10 \%$. We discuss the filling of the ladder gap with impurity states and argue that in the thermodynamic limit the spin ladder with a finite concentration of impurities always shows a Curie susceptibility at low temperatures.
\end{abstract}

PACS numbers: 74.20Hi, 75.10Lp, 71.10+x

Typeset using REVTEX 


\section{INTRODUCTION}

In the last few years spin systems consisting of two interacting spin chains with $S=$ $\frac{1}{2}$, now usually called (two-legged) spin ladders have attracted considerable attention as recently reviewed by Dagotto and Ricel. The spin ladder with isotropic antiferromagnetic interactions exhibits a spin gap in the excitation spectrum which has been shown to be related to the dimer gap (af coupling on the rungs only) as well as to the Haldane gap (strong ferromagnetic coupling on the rungs). It has been concluded that the isotropic spin ladder is in the same phase as the Haldane chain - this can formally be described using matrix product ground states in a generalized spin ladder with additional diagonal bonds .

In the Haldane chain, the investigation of magnetic as well as nonmagnetic impurities has contributed substantially to our understanding of the system: There exist quasifree spins $S=\frac{1}{2}$ at the end of the chain segments created by the impurities as qualitatively predicted by the valence bond ground state 4 and experimentally found in ESR experiments on NENP doped with $\mathrm{Cu}$. that these lead to effects both similar to and different from impurities in the Haldane chain. The essential difference is of geometric origin: impurities in a spin ladder do not break the sequence of magnetic interactions, but create weak links: one spin $S=\frac{1}{2}$ on one leg of the ladder has no counterpart on the other leg and mediates between the adjacent regular ladder structures. In the following we use the term 'unpaired spin' for these spins without counterpart on the opposite leg. A typical configuration with three impurities is shown in fig. 1; neighboring impurities may lead to unpaired spins on the same leg (cis configuration) or on opposite legs (trans configuration). This structural effect that the impurities do not break the coupling along the linear arrangement is analogous to the one of impurities in a chain with nn and nnn interactions - which can actually be considered as a generalized ladder 3 . For a statistical distribution of impurities both the sequence of cis and trans pairs and the distances between these pairs will be statistical. The distance between neighboring unpaired spins is conveniently counted by the number $p$ of complete rungs between them. 
Assuming small impurity concentration $c$ we neglect the possibility of complete breaking of the ladder which is proportional $c^{2}$.

For a microscopic understanding of the effects of a low concentration of defects on the spectrum of the spin ladder we present in the following numerical and analytical calculations for the properties of the ground state and of low-lying excited states of impure ladders with $S=\frac{1}{2}$ and all exchange interactions antiferromagnetic, isotropic and of equal magnitude (which is set equal to unity). In particular we have considered:

- two spins $S=\frac{1}{2}$ in either cis or trans configuration at the edges of an open ladder formed by $p$ rungs (exact diagonalization with the Lanczos algorithm and analytical estimates),

- two impurities at varying distances in either cis or trans configuration in the bulk ladder (exact diagonalization with the Lanzcos algorithm for periodic boundary conditions, DMRG calculations for open boundary conditions and estimates from matrix product states),

- the spin ladder with many impurities (up to six impurities in DMRG calculations and arguments based on the Lieb-Mattis theorem).

Our work is motivated by recent experiments 6 on the ladder material $\mathrm{SrCu}_{2} \mathrm{O}_{3}$ doped with Zn, which show a tendency of the ladder gap to vanish with increasing Zn concentration. Calculations for similar systems which, however, concentrate on somewhat different aspects have recently been done by Motome et all and Martins et al

\section{NUMERICAL METHODS}

We have performed exact diagonalizations using the Lanczos technique for spin ladder systems with two defects and a varying number $p \leq 12$ of complete rungs, i.e. $N=2 p+2$ spins in total and have considered both periodic and open boundary conditions. For open 
boundary conditions we study a ladder with two impurities at the edges of the system and consequently separated by $p$ rungs in either cis or trans configuration. In the case of periodic boundary conditions we fix one defect on the first rung and vary the distance $\left(p_{1}, p_{1} \leq[p / 2]\right.$ rungs) to the second defect. We again consider cis and trans configurations with $p=11$ and $p=12$ rungs. For these configurations we have calculated the energies of the ground state and of low lying excitations and, for open boundary conditions only, the distribution of the magnetization.

For the normal ladder system projection of the Hilbert space on the irreducible representation of the corresponding symmetry groups (reflection and mirror symmetry, total $\mathrm{SU}(2)$ invariance and, in the case of periodic boundary conditions, translational symmetry) drastically reduces the amount of memory for diagonalization within these subspaces. As a conseqence of inserting impurities into the ladder most of these symmetries, in particular translational invariance, are no longer present and the computational effort is correspondingly larger. It is only the $\mathrm{SU}(2)$ invariance of the model which is preserved.

The calculations were performed on a MPP CRAY T3D SC256 of the Zuse Computing Centre Berlin using a parallel implementation of the Lanczos algorithm. The numerical accuracy of these calculations is $10^{-10}$ or better.

Whereas the accuracy of the exact diagonalizations using the Lanzcos approach is high, it can only be done for relatively small systems. To study longer chains we have used the Density Matrix Renormalization Group (DMRG) . Using this method we have studied open ladders from $2 \times 50$ up to $2 \times 100$ spins. As the defects are found to behave like spins localized on the bulk correlation length scale, defects more than 20 sites away from the ladder ends behave effectively like in an infinite system.

As good quantum number we used the total $S^{z}$ spin; in configurations with defects distributed symmetrically around the ladder center, we also used parity. This allows for fast classification of states.

We typically kept $M=100$ to $M=150$ block states. A remark is in order on the precision of the DMRG in this particular application. For the defect-free spin ladder, truncation errors 
are very low, for $M=100 \rho=2.9 \times 10^{-11}$ and for $M=150 \rho=1.1 \times 10^{-12}$, corresponding to an extremely small error in the energies of the defect-free system for low-energy states. The effective precision of the DMRG is however greatly reduced, when a defect or defect pair are added. The block states from the step before are not optimally chosen to represent the altered system. We find that the introduction of defects effectively reduces the precision of the DMRG by up to several orders of magnitude.

This problem becomes particularly pressing in defect configurations nonsymmetric with respect to the ladder center. For any even number of defects, the ground state is the lowest eigen state in the $S^{z}=0$ sector. A nonsymmetric defect configuration implies that during the growth process there will be an odd number of defects present in some DMRG steps. The total number of spins on the ladder changes from even to odd. Consequently, the ground state jumps to the $S^{z}= \pm \frac{1}{2}$ sector, to which the available block states are not well adapted, drastically reducing the precision of the DMRG. However, the error can be greatly reduced by using the finite size DMRG algorithm 10 11: The whole chain is recalculated in the presence of the complete defect configuration without any jumps between different $S^{z}$ sectors. The gain in precision by far exceeds the one obtained by increasing $M$.

To illustrate this point, let us consider the case of a $2 \times 50$ spin ladder with two defects in trans-configuration on rungs 24 and 26. We have calculated the lowest eigenstates with $S^{z}=0$ and $S^{z}=1$, keeping $M=40$ and $M=100$ states. In Table [ the energies given are for the chain calculated by conventional DMRG, and after one resp. two iterations applying the finite size algorithm. Obviously, even for $M=100$ the unmodified DMRG (first line) gives results of the order of 0.01 away from the converged result, whereas the finite size algorithm produces highly precise results even for $M=40$ (compare results in second and third lines).

All DMRG calculations were performed on a PentiumPro 200MHz machine running under Linux. 


\section{TWO UNPAIRED SPINS}

In this section we discuss the spectra and spin configurations for two unpaired spins in ladders with both open and periodic boundary conditions, presenting and comparing numerical results from both Lanczos and DMRG calculations and from analytical approaches.

\section{Numerical results for the spectrum of unpaired edge spins}

We consider the configuration with $p$ rungs connecting two spins $\vec{S}, \vec{S}^{\prime}$ in either cis or trans configuration at the ends. Using the Lanczos algorithm we have exactly diagonalized systems with $p \leq 12$. We find that there is an energy gap of the order of the known ladder gap $(\Delta \approx 0.53)$ but the ground state is splitted into a singlet and a triplet state. As a consequence of the Lieb-Mattis theorem the lowest state for our configuration is a singlet if the two unpaired spins are on different sublattices ( $p$ even for cis spins and $p$ odd for trans spins) and a triplet if they are on the same sublattice ( $p$ odd for cis spins and $p$ even for trans spins) as discussed in ref. 12. We interpret this splitting as resulting from an effective interaction between the unpaired spins $\vec{S}, \vec{S}^{\prime}$ at the ladder boundaries and we derive an effective coupling by writing a Hamiltonian in the subspace of these lowest two states as

$$
H_{ \pm}^{(p)}=E_{0, \pm}+J_{e f f, \pm}^{e d g e}(p) \vec{S} \vec{S}^{\prime}
$$

The index \pm refers to the sign of $J_{\text {eff }}$, i.e. antiferromagnetic and ferromagnetic effective

interaction respectively. $J_{e f f}^{e d g e, \pm}(p)$ is given in Table $\mathbb{I}$ and is also plotted in fig. 2 to show its dependence on $p . J_{e f f, \pm}^{e d g e}$ actually is identical to the singlet-triplet splitting of the ground state. An excellent fit for $p \geq 5$ is obtained as

$$
\begin{aligned}
E_{0,+}(p) & =-1.157 p, \\
J_{\text {eff }, \pm}^{\text {edge }}(p) & =J_{0, \pm} e^{-p / \xi}, \quad \text { with } \quad J_{0,+} \approx 0.674, \quad J_{0,-} \approx 0.714, \quad \xi \approx 3.1 .
\end{aligned}
$$

The uncertainty in these data as determined from a least square fit is $10^{-6}$ for $J_{0, \pm}$ and better for $E_{0,+}$. Due to the finite size of the open chain, $E_{0,-}(\approx-1.161 p)$ cannot be determined 
with equal accuracy. We draw attention to the fact that the best fit is purely exponentially decaying and that a behavior $\propto \exp (-p / \xi) / \sqrt{p}$ can be excluded. This is in parallel to the behavior of an open Haldane chain 9 .

\section{Spectrum of unpaired spins in the bulk ladder: Numerical results}

Contrary to the Lanczos approach the DMRG allows to deal with sufficiently long ladders so that the two unpaired spins can be at some rather large distance and at the same time sufficiently far from the boundaries to identify their bulk interaction. The splitting of the ground state for two unpaired spins is again described by a Hamiltonian as above with $J_{e f f, \pm}^{e d g e}(p)$ replaced by $J_{e f f, \pm}^{b u l k}(p)$. The latter quantity is also given in Table $\llbracket$ and shown in fig. 2. We find (for $p \geq 5$ )

$$
J_{\text {eff } f \pm}^{\text {bulk }}(p)=J_{0, \pm}^{\text {bulk }} e^{-p / \xi}, \quad \text { with } \quad J_{0,+}^{\text {bulk }} \approx 0.43, \quad \xi \approx 3.1
$$

$J_{0,-}^{b u l k}$ is approximately equal to $J_{0,+}^{b u l k}$, but determined less accurately. We notice that $J_{e f f}^{b u l k}(p)$ is reduced with respect to $J_{\text {eff }}^{\text {edge }}(p)$ by a reduction factor $r_{\text {exc, }, \text {, }}$

$$
J_{e f f, \pm}^{b u l k}(p)=r_{e x c, \pm}(p) J_{e f f, \pm}^{e d g e}(p) .
$$

For effectively antiferromagnetic interaction the reduction factor $r_{e x c}$ is $p$ independent, $r_{e x c} \approx 0.65$, for effectively ferromagnetic interaction it approaches this value asymptotically. This reduction will be explained quantitatively below; qualitatively it is due to the fact that the unpaired spin projection delocalizes both into its right and left neighborhood in the bulk case whereas there are neighbors on one side only in the boundary case. The dependence of the effective exchange on distance is again purely exponential and the prediction in ref. 12, which includes an additional factor $1 / \sqrt{p}$ is not verified from our data. The 'best' fit enforcing the factor $1 / \sqrt{p}$ ends up with $\xi \approx 4$, i.e. a correlation length which is not appropriate.

Using the Lanczos algorithm, we have also done exact diagonalizations for configurations with periodic boundary conditions (pbc) and two spins $\vec{S}, \vec{S}^{\prime}$ in cis or trans configuration 
separated by $p_{1}$, resp. $p_{2}$ rungs. The spectra are shown in fig. $3 \mathrm{a}$, b for $p_{1}+p_{2}=12$ and in fig. 3 $\mathrm{c}$, d for $p_{1}+p_{2}=11$; their qualitative structure is the same as for the open ladder. We find that the smaller of the two values $p_{1}, p_{2}$ determines whether the ground state is singlet or triplet when the rules given above for the open ladder are used. From the graphs presented in fig. Bit is also evident that a much larger singlet-triplet splitting of the ground state is obtained for $p_{1}+p_{2}$ even than for $p_{1}+p_{2}$ odd. The obvious reason is that the two exchange interactions which have to be added for pbc have equal (different) sign for $p_{1}+p_{2}$

even (odd). Using again the concept of an effective exchange interaction, $J_{\text {eff }}^{p b c}\left(p_{1}, p_{2}\right)$, we expect for two unpaired spins (resulting from two impurities in a periodic ladder)

$$
J_{\text {eff }}^{p b c}\left(p_{1}, p_{2}\right)=J_{\text {eff }}^{\text {bulk }}\left(p_{1}\right)+J_{\text {eff }}^{\text {bulk }}\left(p_{2}\right)
$$

Inserting the results as given before, we find that this relation is obeyed to within $10 \%$.

In fig. 3 we also show the results for the lowest excited states, i.e. in particular the splitting of the regular ladder gap with energy $\approx \Delta$. For most of the defect configurations the effective interactions have different sign for the ground state and for the first excited state (energy of the regular ladder gap). For example, a singlet ground state is related to a triplet as the lower one of the states of energy $\approx \Delta$, as follows from a simple coupling of the impurities to the lowest bulk excitation with $S=1$. This rule, however, is not strictly obeyed and a detailed discussion of the structure of the excited states in the impure ladder has to be reserved for future work.

\section{Analytical approaches to unpaired spins on the ladder edges}

A qualitative understanding of our numerical results for the open ladder can be obtained using matrix product wave functions as described in ref. 3. We consider a system with two spins at the ladder edges in trans configuration and $p$ rungs in between; A wave function describing this system with four degrees of freedom can be written down as a $2 \times 2$ matrix product (MP) wave function, 


$$
|\psi\rangle_{\sigma, \sigma^{\prime}}=\left[\prod_{j=1}^{p+1} g_{j}\right]_{\sigma, \sigma^{\prime}}, \quad g_{j}=\left(\begin{array}{cc}
a\left|t_{0}\right\rangle_{j}+b|s\rangle_{j} & -a \sqrt{2}\left|t_{+}\right\rangle_{j} \\
a \sqrt{2}\left|t_{-}\right\rangle_{j} & -\left(a\left|t_{0}\right\rangle_{j}-b|s\rangle_{j}\right)
\end{array}\right)
$$

Here the matrix $g_{j}$ describes the coupling of spins situated on diagonal sites on two adjacent rungs to singlets, resp. triplets; the boundary spins are coupled likewise to the neighboring rung. The value of $b^{2}=1-3 a^{2}$ is determined by minimization of the energy; for the numerical estimates below we take the result for the infinite ladder $3, b \approx 0.1735$. We have chosen the two edge spins in trans configuration where a MP wave function is easily written down, whereas this is more difficult for two spins in cis configuration (with e.g. two more spins on the upper leg than on the lower leg); the latter case requires a more detailed analysis which will be published separately. Instructive limiting cases of the wavefunction of eq.(6) are:

- For $a=b=\frac{1}{2}$ it is the wave function for $p$ singlets on the $p$ rungs and truly free boundary spins $\vec{S}, \vec{S}^{\prime}$. This wave function actually is an eigenfunction to the MajumdarGhosh Hamiltonian for a finite chain with $2 p$ spins and one additional free spin at each open end. Their spin projections can be identified with the matrix indices $\sigma, \sigma^{\prime}$.

- For $b=0, a=1 / \sqrt{3}, \quad|\psi\rangle_{\sigma, \sigma^{\prime}}$ describes $p+1$ units with $S=1$ on the diagonals of the ladder, i.e. it is identical to the 4 eigenfunctions for the AKLT chain with open endst. In this limit it is known that at the edges there exist quasifree $S=\frac{1}{2}$ spins which extend somewhat into the bulk of the ladder. Note that the matrix indices $\sigma, \sigma^{\prime}$ now can no more be identified with the spin projections of the edge spins.

- The general case will be intermediate and the boundary spins extend into the adjacent bulk ladder to an extent determined by the correlation length. This correlation length is underestimated by the MP ansatz but the tendency of the variation is given correctly: it decreases with increasing $b$, i.e. from the AKLT limit via the ladder configuration to the Majumdar Ghosh limit. 
For a more quantitative treatment we couple the $4 \mathrm{MP}$ wavefunctions $\left[\prod_{j} g\right]_{\sigma, \sigma^{\prime}}, \sigma, \sigma^{\prime}=$ \pm 1 , to singlet, resp. triplet states and calculate the effective exchange energy from the energy difference of these states. This leads to the following results for a configuration with $p$ complete rungs in between:

$$
J_{\text {eff,MP }}^{\text {edge }}(p)= \pm J_{0} e^{-p / \xi}, \quad \text { with } \quad J_{0} \approx 0.77, \quad \xi^{-1} \approx 1.23
$$

This gives the correct sign of $J_{\text {eff }}$ (i.e. ferro- or antiferromagnetic if $\sigma, \sigma^{\prime}$ are on the same or on different sublattices respectively) and a remarkably good numerical value for $p=0$; the decay with distance, however, is too strong, due to the fact that the correlation length is underestimated in MP states.

We notice that the low energy structure of the spectrum is the generalization to spin ladders of the Kennedy triplet observed in Haldane chains with open boundary conditions 13 : The latter is obtained (in the AKLT limit) by taking $b \rightarrow 0$ in our wavefunction. In the ladder the wavefunction is more general than just a RVB ansatz, but the effect of the quasifree boundary spins is the same. In this sense our results are related to the results of Hida 14 who investigated the ladder with ferromagnetic coupling $\lambda$ on the rungs. This coupling connects two spins which in our antiferromagnetic ladder are not coupled directly but as nnn by two af bonds; from our calculations we see that this leads to the same effects.

An alternative approach to arrive at a theoretical estimate for $J_{e f f, \pm}^{e d g e}$ is to integrate out the spin degrees of freedom on the rungs between the boundary spins. For this purpose we write the general Hamiltonian with boundary spins $\vec{S}_{0}$ and $\vec{S}_{2 p+1}$ as

$$
H=H_{\text {ladder }}(1,2 \ldots 2 p)+h_{0,1}+h_{2 p, 2 p+1}
$$

We eliminate $h_{0,1}$ and $h_{2 p, 2 p+1}$ to first order by a suitable canonical transformation and average over the eigenstates of the complete rungs forming the intermediate part of the ladder to end up with an effective low-energy Hamiltonian of the form

$$
H_{ \pm}^{(p)}=E_{0, \pm}(p)+J_{e f f, \pm}(p) \vec{S}_{0} \vec{S}_{2 p+1}
$$


The explicit results for two and three intermediate rungs between the boundary spins are

$$
\begin{aligned}
& E_{0, \pm}(2)=-\frac{5}{16}, \quad J_{\text {eff },+}^{\text {edge }}(2)=\frac{1}{3}, \quad J_{\text {eff },-}^{\text {edge }}(2)=-\frac{1}{4} \\
& E_{0, \pm}(3)=-0.344, \quad J_{\text {eff },+}^{\text {edge }}(3)=-0.2377, \quad J_{\text {eff },-}^{\text {edge }}(3)=0.2248 .
\end{aligned}
$$

These results agree to the numerical data within 20\%. For two isolated spins, higher order contributions to the canonical transformation will change the numbers towards the correct values but due to isotropy and to the fact that we are dealing with $S=\frac{1}{2}$ the form of the Hamiltonian will not be affected. Although this approach gives only rough estimates, it serves to illustrate that for more than two unpaired spins higher order terms in the canonical transformation will lead to pair interactions between unpaired spins which are not nearest neighbors and to $m$-spin interactions $(m \geq 4)$. The difference between the above analytical result and the numerical results should be considered as an indication that these higher spin interactions cannot be neglected at the outset and a description of the impure chain in terms of an effective pair Hamiltonian for unpaired spins as in ref. 12 needs justification. We will return to this question in section IV.

\section{Spin configurations in the presence of two unpaired spins}

We now discuss in more detail the spin configuration in the presence of two unpaired spins separated by a number of rungs larger than the correlation length. Then the spin projection will spread into the adjacent part of the ladder, i.e. into the rungs to the right and to the left of its site (or into the rungs on one side only in the case of edge spins). In

order to demonstrate this effect for an unpaired spin with a given projection $\left\langle S^{z}\right\rangle= \pm \frac{1}{2}$ we consider the quantity $\left\langle S_{m, \pm}^{z}\right\rangle$, where $m$ is the distance from the unpaired spin $(m=0$ denotes the unpaired spin) and \pm distinguishes between spins on the same $(+)$, resp. opposite $(-)$, sublattice as the unpaired spin. When the two unpaired spins are sufficiently separated we obtain a clear picture of the situation in the neighborhood of a single unpaired spin coupling the two unpaired spins to $S_{t o t}=1$ and considering states with $S_{t o t}^{z}=+1$. Results from the 
DMRG and Lanczos which illustrate this redistribuiton of the spin projection are shown in

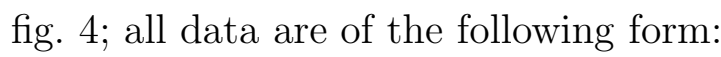

$$
\begin{array}{rlrl}
\left\langle S_{m, \alpha}^{z}\right\rangle & =s_{\text {center }}^{z} \text { for } m=0 \\
& =s_{\text {tail }}^{z} e^{-\frac{|m|}{\xi}} \quad \text { for } \quad & |m|>0, \alpha=+ \\
& =-s_{\text {tail }}^{z} e^{-\frac{|m|}{\xi}}+\delta_{m} \quad \text { for } \quad|m|>0, \alpha=-
\end{array}
$$

When the two unpaired spins are sufficiently far apart, the total spin projection $+\frac{1}{2}$ has to be recovered, i.e.

$$
s_{\text {center }}^{z}+2 \sum_{m=1}^{\infty} \delta_{p}=\frac{1}{2}
$$

for an unpaired spin in the bulk, and

$$
s_{\text {center }}^{z}+\sum_{m=1}^{\infty} \delta_{p}=\frac{1}{2}
$$

for an unpaired spin at the edge. $\delta_{p}$ is a correction which approaches zero rapidly for $p>5$. Quantitative results from the two approaches are:

For an unpaired spin in the bulk (from DMRG):

$$
\xi=3.1 \quad s_{\text {center }}^{z}=0.294 \quad s_{\text {tail }}^{z}=0.247,
$$

and for an unpaired spin at the edge (from Lanczos, after corrections for the finite size of the system, i.e. the contributions of the second impurity):

$$
\xi=3.1 \quad s_{\text {center }}^{z}=0.348 \quad s_{\text {tail }}^{z}=0.308 .
$$

The amplitude $s_{\text {tail }}^{z}$ characterizes the redistribution of spin projection into the adjacent rungs and is seen to be rather large. The total excess spin on the positive sublattice is 0.894 (bulk), resp. 1.117 (edge) and on the negative sublattice -0.792 (bulk), resp. -0.965 (edge). We notice that the asymptotic exponential behavior governed by the correlation length is 
correct for all sites on the sublattice of the unpaired spin whereas it is approached within typically 5 rungs on the opposite sublattice.

The numbers given above are consistent with the difference between the effective exchange constants $J^{e d g e}$ and $J^{b u l k}$ noticed above and give a microscopic understanding of this difference as is seen in the following way: For an unpaired spin in the bulk of the ladder the magnitudes of both $s_{\text {center }}^{z}$ and $s_{\text {tail }}^{z}$ are reduced by a factor of $\approx .8$ as compared to an unpaired spin at the ladder edge. Since the effective interaction of two unpaired spins will be determined by the tails of the spin distribution, $J_{\text {eff }}$ will be smaller for unpaired spins in the bulk ladder by

$$
\left(\frac{s_{\text {tail }}^{z, \text { bulk }}}{s_{\text {tail }}^{z, e d g e}}\right)^{2} \approx 0.64
$$

consistent with the value $r_{\text {exc }} \approx .65$ found from the analysis of the energy levels above.

A redistribution of $S^{z}$ in the neighborhood of an unpaired edge spin is also obtained from the MP wave function above which gives

$$
s_{\text {center }}^{z}=a^{2}+a b \approx 0.422 .
$$

The behavior on the adjacent rungs is different in detail from the numerical results: $S_{p}^{z}$ decays with alternating sign and purely exponential with different amplitudes on the leg of the unpaired edge spin $\left(\tilde{s}_{\text {tail }}^{z}=a^{2}+a b \approx 0.422\right)$ and on the opposite leg $\left(\tilde{s}_{\text {tail }}^{z}=\right.$ $-a /(a+b) \approx-0.767)$. Again, the MP approach gives a reasonable qualitative picture but fails quantitatively.

\section{MANY IMPURITIES}

It is natural to assume that the results obtained for two unpaired spins can be generalized to give results for the low energy properties of the general impure ladder with impurity concentration $c$ by using pair exchange interactions between unpaired spins which are nearest neighbors only and determining sign and magnitude of these interactions from the results 
in section III. For a random distribution of impurities this means that the impure ladder reduces to a spin chain with exchange constants which are random with respect to both sign and magnitude. This is the model used in ref. 12 .

In this section we will investigate this assumption more closely by comparing exact (DMRG) spectra for various configurations with four and six impurities to spectra obtained from the assumption above. We have restricted ourselves to an even number of impurities in order to facilitate the comparison between different impurity concentration (for an odd number of impurities the spin of the ground state will be half integer). A similar approach to the case of the spin-Peierls substance $\mathrm{CuGeO}_{3}$ with impurities has been done in ref. 88.

Before presenting these numerical results we want to discuss some aspects of the general impure ladder with antiferromagnetic exchange interactions only and $L$ rungs, i.e. $2 L$ sites, of which $N$ sites are occupied by nonmagnetic impurities. The ground state of the remaining $2 L-N$ spins $S=\frac{1}{2}$ can take values $S_{\text {tot }}$ of total spin between 0 and $N / 2$, depending on the distribution of the impurities on the two sublattices. For a random distribution of the impurities the distribution of $S_{t o t}$ is easily obtained from the theorem of Lieb and Mattis which states $S_{t o t}=\frac{1}{2}\left|N_{A}-N_{B}\right|$, where $N_{A}, N_{B}$ are the numbers of defects on the $A$ and $B$ sublattices respectively (for configurations with two defects on the same rung, which break the ladder, this gives $S_{\text {tot }}$ only for one out of a number of degenerate ground states). Thus for a random distribution of defects the probability $g\left(S_{t o t}\right)$ for a ground state to have total $\operatorname{spin} S_{t o t}$ is

$$
\begin{array}{lll}
\text { for } \quad S_{t o t}=0 & g\left(S_{t o t}\right)=\left(\begin{array}{c}
N \\
N / 2
\end{array}\right) \\
\text { for } \quad S_{t o t}>0 & g\left(S_{t o t}\right)=2\left(\begin{array}{c}
N \\
N / 2-S_{t o t}
\end{array}\right) .
\end{array}
$$

For $N \gg 1$ we obtain from Stirlings formula the relative weight of states with $S_{t o t}=0$ and $S_{t o t}=1\left(2^{N}\right.$ is the total number of defect induced states)

$$
\frac{g\left(S_{t o t}=0\right)}{2^{N}}=\sqrt{\frac{2}{\pi N}}, \quad \frac{g\left(S_{t o t}=1\right)}{2^{N}}=2 \sqrt{\frac{2}{\pi N}} .
$$

The ground state spin value with largest probability therefore is $S=1$. Of particular 
interest are the average values of the ground state spin for a random distribution of $N \gg 1$ impurities. They are calculated using eq. (16) to give:

$$
\begin{aligned}
& \left\langle S_{t o t}\right\rangle=\sqrt{\frac{N}{2 \pi}} \\
& \left\langle S_{t o t}^{2}\right\rangle=\frac{N}{4}
\end{aligned}
$$

Thus the result obtained in ref. 12 from a random walk argument is seen to be an exact consequence of the Lieb-Mattis theorem. For a discussion of the low temperature susceptibility in some given defect configuration we use $S_{0}$ to denote the total spin of the ground state (with zero energy) and characterize the remaining states $\alpha=1,2 \ldots \alpha_{m}=2^{N}-1$ by their total spin $S_{\alpha}$ and excitation energy $\Delta_{\alpha}>0$. The limiting susceptibility for low magnetic fields is then given by

$$
\chi=\frac{\left(g \mu_{B}\right)^{2}}{3 k_{B} T} \frac{z\left(S_{0}\right)+\sum_{\alpha=1}^{\alpha_{m}} z\left(S_{\alpha}\right) e^{-\beta \Delta_{\alpha}}}{\left(2 S_{0}+1\right)+\sum_{\alpha=1}^{\alpha_{m}}\left(2 S_{\alpha}+1\right) e^{-\beta \Delta_{\alpha}}},
$$

with

$$
z\left(S_{\alpha}\right)=2 S_{\alpha}\left(S_{\alpha}+\frac{1}{2}\right)\left(S_{\alpha}+1\right)
$$

If the ground state of the impure ladder is not a singlet, i.e. $S_{0}>0$, leading to $z\left(S_{0}\right)>0$, a Curie susceptibility results in the low temperature limit. For $S_{0}=0$, leading to $z\left(S_{0}\right)=0$, the susceptibility shows an activated temperature dependence characteristic of a gapped system $\left(\Delta_{1}\right.$ finite); if the low-lying states get dense with an asymptotic density of states $\rho(\epsilon) \sim \epsilon^{-\alpha}$ the low temperature behavior of the susceptibility changes to $\chi(T) \sim T^{-\alpha}$. In particular a temperature independent susceptibility results for $\alpha=0$, i.e. a constant density of states (this is the case of the $S=\frac{1}{2}$ Heisenberg antiferromagnetic chain - actually it should be realized in this context that a concentration of $c=\frac{1}{4}$ is sufficient to turn the ladder geometry into that of a single chain). All these deviations from a Curie behavior require a singlet ground state, which according to eq.(17) occurs with negligible weight in the limit of a macrosocpic system. We therefore conclude that the low temperature susceptibility of the impure ladder always follows a Curie law. This confirms from a different point of view the result $\alpha=1$ which has been obtained from a renormalization group approach 15 . 
When the limiting value of the Curie constant for $T \rightarrow 0$ is calculated on the basis of the above expressions, we find that due to the random positions of the defects the factor $\frac{3}{4}$ (resulting from $S(S+1)$ for $S=\frac{1}{2}$ ) is replaced by $\frac{1}{4}$. The experimentally interesting behavior at finite temperatures involves the transition between these two limiting cases, i.e. a change in Curie constant by a factor of 3 . It will be determined to a large extent by the density of states. This is not easily accessible, and in the remainder of this section we present what can be learned from numerical calculations.

In fig. 5 and table [II we present a number of spectra with levels classified according to total spin $S$ and corresponding results from the effective pair model. We have chosen the parameters under the following aspects:

1. We want to control the effective model,

2. we want to illustrate the filling of the gap with defect states, and

3. we want to present the effects of the different sign combinations for defects on a given sequence of rungs.

For the comparison between the complete ladder spectra as obtained from the DMRG and the spectra of the effective model we refer to table [II]. We see that the agreement is very satisfying, deviations are generally below $10 \%$ with a tendency of better accuracy for low energies. We note that all DMRG energy spectra overestimate the true energies. Further corrections will therefore rather improve the agreement between effective model and DMRG calculations.

Since the spectra obtained by the two approaches agree so well, in fig. 5 DMRG spectra (full lines) only are given when these are available. However, the higher singlets and triplets were not accessible at reasonable computational expense as the respective states are already quite high-lying in the $S_{\text {tot }}^{z}=0,1$ sectors. It is only in these cases that we have included into fig. 5 the spectra obtained in the effective model (light gray lines). 
In fig. 5(a-e) we show spectra for four unpaired spins with 4,3 and 5 complete rungs in between. The possible combinations of the signs are seen to lead to quite different spectra and the general tendency to fill the regular ladder gap is evident. Figs. 5(a, e) result from each other qualitatively by an overall sign change. Although the magnitudes of the interactions are somewhat different (compare table III) this is clearly evident in these spectra. We therefore present only three out of the remaining six spectra for this combination of distances in fig. 5(c - e). Fig. 5(f, g) presents two examples of symmetric configurations of four spins (here levels are characterized by the additional quantum number parity which we have not indicated). The six-defect spectrum shown in fig. 5(h) again is for a symmetric configuration. In particular these data illustrate, beyond related calculations, which have been done for the case of two impurities 7 , how the density of states with $S=1$ may increase at low energies with the number of impurities. However, a much larger number of impurities will be required to obtain a reliable numerical estimate for the low energy density of states $\rho(\epsilon)$.

\section{CONCLUSIONS}

We have demonstrated that interacting impurities drastically change the low-energy spectrum of an antiferromagnetic spin ladder. A statistical distribution of impurities reduces the ladder, as far as its low energy spectrum is concerned (energy range of the pure ladder gap), to a $S=\frac{1}{2}$ chain with random interactions, a model which has recently been used to discuss the expected low temperature properties of the impure ladder 12 . From our calculations precise information on the parameters of the effective interactions is available. For large distances between defects the effective coupling strength is found to decay purely exponentially. We have found that it is sufficient to use two spin interactions to describe the spectrum of the effectively random chain to within $10 \%$. The spectra which we have calculated also illustrate the beginning of the process that a random distribution of impurities with concentration $c$ produces a large number (of the order of $2^{c L}$ ) of low-lying states which 
will fill the ladder gap as observed experimentally6. Applying the Lieb-Mattis theorem to the impure ladder with antiferromagnetic interactions only we argue that for $T \rightarrow 0$ the susceptibility is characterized by a $1 / T$ divergence with finite Curie constant. Our numerical results, however, are not sufficiently accurate to draw conclusions about the change in Curie constant with increasing temperature.

From our results it becomes also clear that interactions between unpaired spins which are not nearest neighbors and $m$-spin interactions are present and cannot be neglected in the effective model at the outset. They have, however, turned out not quantitatively relevant for the low-energy spectra which we have computed.

The effects of impurities on the spin distribution which we have presented show some similarity to what has been discussed in the context of impure Haldane chains 5 and it is useful to qualitatively compare these two phenomena once more: In both systems the unpaired spin at the position of the impurity shares its magnetic moment with the adjacent part of the system - and the degree of this mixing, i.e. its spatial extent, is determined by the correlation length. In the Haldane case, one deals with a physically different magnetic impurity spin; it interacts with that part of the unpaired boundary spin which remains localized, leading to the experimentally observed splitting of the ESR spectra. In the spin ladder a nonmagnetic impurity is present and the corresponding unpaired spin shares its magnetic moment with the adjacent parts of the ladder; the tails of this magnetic moment introduce an effective interaction leading to the experimentally observable splitting of the low-energy spectrum.

\section{ACKNOWLEDGEMENTS}

This work was supported by the German Federal Ministry of Research and Technology (BMBF) under contract number 03-MI4HAN-8. We wish to thank Regionales Rechenzentrum Niedersachsen and Zuse Rechenzentrum Berlin for their helpful cooperation with part of the numerical calculations. 


\section{REFERENCES}

${ }^{1}$ E. Dagotto and T.M. Rice, Science 271, 618 (1996).

${ }^{2}$ S.R. White, Phys. Rev. B 53, 52 (1996).

${ }^{3}$ S. Brehmer, H.-J. Mikeska and U. Neugebauer J.Phys.: Condensed Matter, in print

${ }^{4}$ I. Affleck, T. Kennedy, E. Lieb and H. Tasaki, Phys. Rev. Lett. 59, 799 (1987); Commun. Math. Phys. 115, 583 (1988).

${ }^{5}$ M. Hagiwara, K. Katsumata, I. Affleck, B.I. Halperin and J.P. Renard, Phys. Rev. Letters 65, 3181 (1990)

${ }^{6}$ M. Azuma, Y. Fujishiro, M. Takano, T. Ishida, K. Okuda, M. Nohara and H. Tagaki, preprint (1996)

${ }^{7}$ Y. Motome, N. Katoh, N. Furukawa and M. Imada, J. Phys. Soc. Jpn. 65, No.7 (1996);

N. Katoh, Y. Motome, N. Furukawa and M. Imada, preprint (1996)

${ }^{8}$ G.B. Martins, E. Dagotto and J.A. Riera, preprint (1996)

${ }^{9}$ S.R. White, Phys. Rev. Lett. 69, 2863 (1992)

${ }^{10}$ S.R. White, Phys. Rev. B 48, 10345 (1993)

${ }^{11}$ U. Schollwöck and T. Jolicoeur, Europhys. Lett. 30, 493 (1995)

${ }^{12}$ M. Sigrist and A. Furusaki, preprint

${ }^{13}$ T. Kennedy, J.Phys.: Condensed Matter 2, 5737 (1990)

${ }^{14}$ K. Hida, J. Phys. Soc. Jpn. 64, 4896 (1995)

${ }^{15}$ E. Westerberg, A. Furusaki, M. Sigrist and P.A. Lee, Phys. Rev. Letters 75, 4302 (1995) 


\section{FIGURES}

FIG. 1. Structure of a spin ladder with three impurities leading to unpaired spins in cis resp. trans configuration.

FIG. 2. Effective exchange interactions for two unpaired spins at the edges and in the bulk of a spin ladder. Exchange energies for effective ferromagnetic and antiferromagnetic interactions are plotted separately.

FIG. 3. Low-lying energy levels for ladders with periodic boundary conditions and two unpaired spins with a distance of $p_{1}$ resp. $p_{2}$ complete rungs. Spectra for unpaired spins with effective antiferromagnetic, resp. ferromagnetic, interactions are shown separately. (a, b) $p_{1}+p_{2}=12$ rungs, $(\mathrm{c}, \mathrm{d}) p_{1}+p_{2}=11$.

FIG. 4. Distribution of the excess z-component of spin (magnitude 1/2) resulting from the unpaired spin opposite to an impurity into the adjacent rungs of the ladder (the positive and negative contributions for each rung $p$ are alternating between the two legs).

FIG. 5. Low energy spectra of the ladder with four and six impurities from DMRG (black lines) and from the effective model (light gray lines, only when DMRG data are not available) for different configurations characterized by the sign $(\mathrm{F}, \mathrm{AF})$ and the number of complete rungs between the unpaired spins as indicated in the graphs. (a) - (g) four unpaired spins: (a) F4 - F3

- F5, (b) AF4 - F3 - AF5, (c) F4 - AF3 - AF5, (d) F4 - F3 - AF5, (e) AF4 - AF3 - AF5, (f) F5 AF4 - F5, (g) F3 - AF4 - F3; (h) six unpaired spins: AF5 - F4 - AF6 - F4 - AF5. 


\section{TABLES}

TABLE I. Energies calculated by the DMRG before (first line) and after one (second line) and two (third line) applications of the finite size algorithm.

TABLE II. Effective exchange energies for two unpaired spins at the edge and in the bulk of a ladder with $p$ rungs in between. Two energies are given for each $p$, corresponding to the two positions on each rung.

TABLE III. Low energy spectra of the ladder with four and six impurities for different configurations as in fig.5: comparison of DMRG and effective Hamiltonian results. 
Fig. 1

Trans configuration i Trans configuration

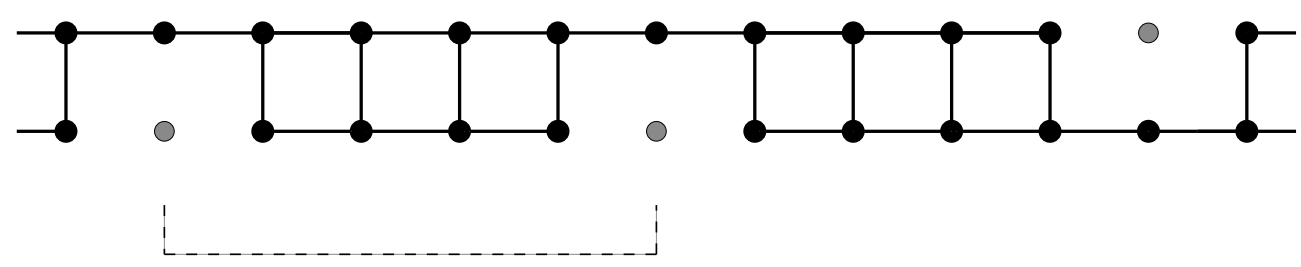

Cis configuration 
Fig. 2

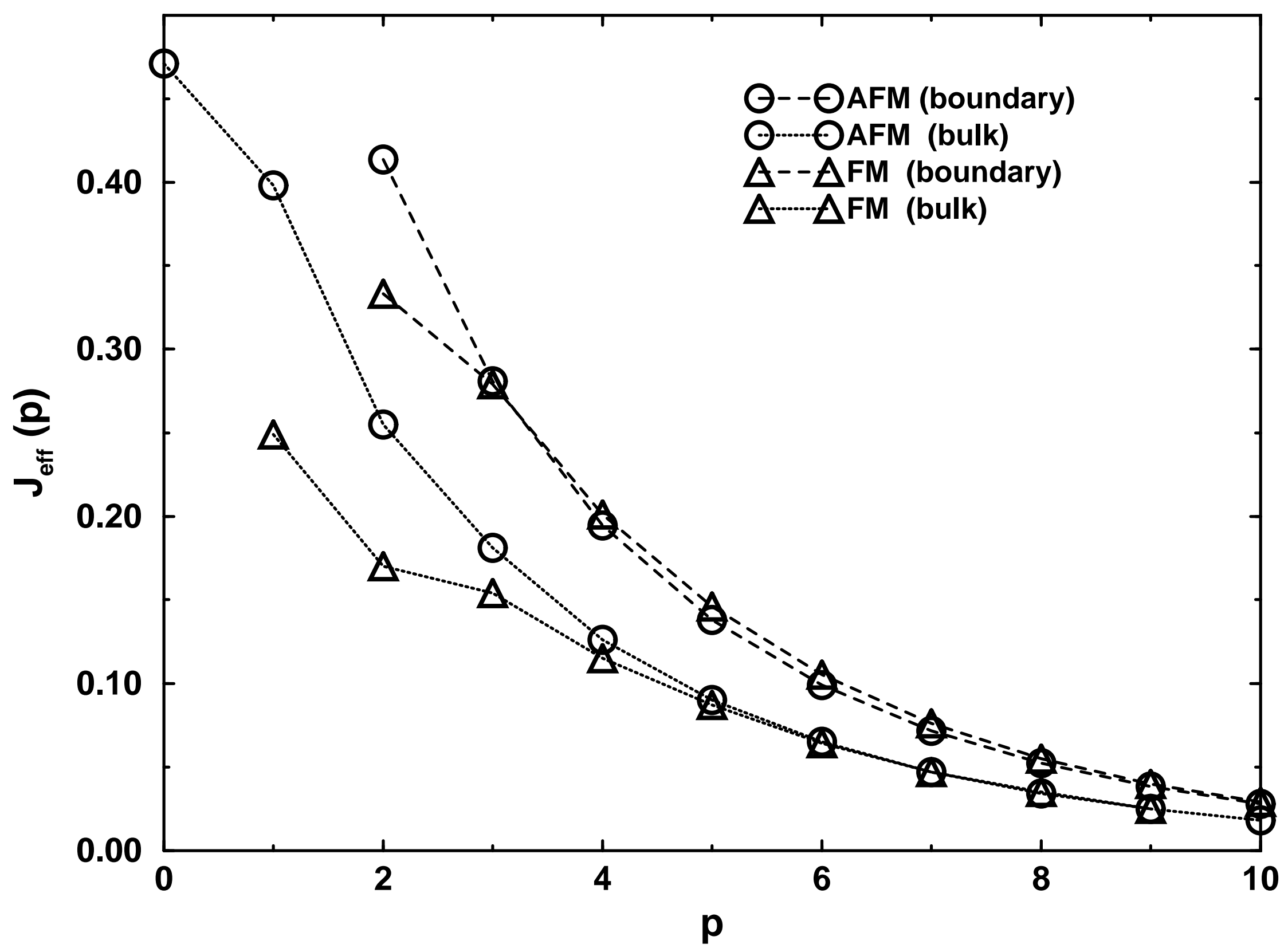


Table 2

\begin{tabular}{|c|c|c|}
\hline \multicolumn{3}{|c|}{$J_{e f f}(\mathrm{ED})$} \\
\hline rungs (n) & antiferromagnetic & ferromagnetic \\
\hline 2 & 0.4134342076 & 0.3332389325 \\
\hline 3 & 0.2808007550 & 0.2790132929 \\
\hline 4 & 0.1944368705 & 0.2010576140 \\
\hline 5 & 0.1379125645 & 0.1459181428 \\
\hline 6 & 0.0990135730 & 0.1051339920 \\
\hline 7 & 0.0717149472 & 0.0759464504 \\
\hline 8 & 0.0522047020 & 0.0549482466 \\
\hline 9 & 0.0381181864 & 0.0398361954 \\
\hline 10 & 0.0278774960 & 0.0289263601 \\
\hline 11 & 0.0204037286 & 0.0210324667 \\
\hline 12 & 0.0149374382 & 0.0153089483 \\
\hline \multicolumn{3}{|c|}{$J_{e f f}(\mathrm{DMRG})$} \\
\hline rungs (n) & " antiferromagnetic & "ferromagnetic \\
\hline 0 & 0.471 & \\
\hline 1 & 0.398 & 0.249 \\
\hline 2 & 0.255 & 0.170 \\
\hline 3 & 0.181 & 0.154 \\
\hline 4 & 0.126 & 0.115 \\
\hline 5 & 0.090 & 0.087 \\
\hline 6 & 0.065 & 0.064 \\
\hline 7 & 0.047 & 0.047 \\
\hline 8 & 0.034 & 0.035 \\
\hline 9 & 0.025 & 0.025 \\
\hline 10 & 0.018 & \\
\hline
\end{tabular}


Fig. 3.a

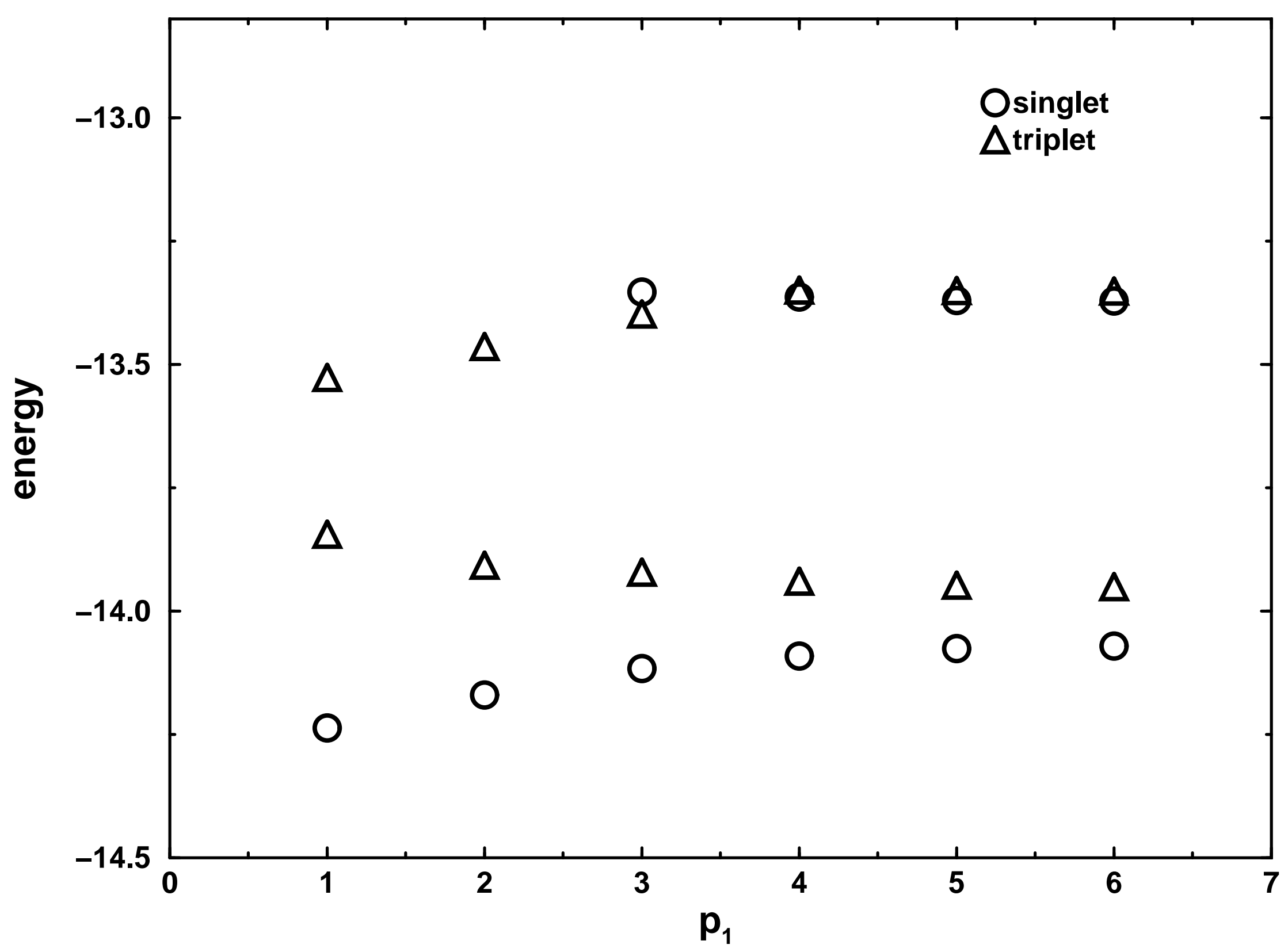


Fig. 3.b

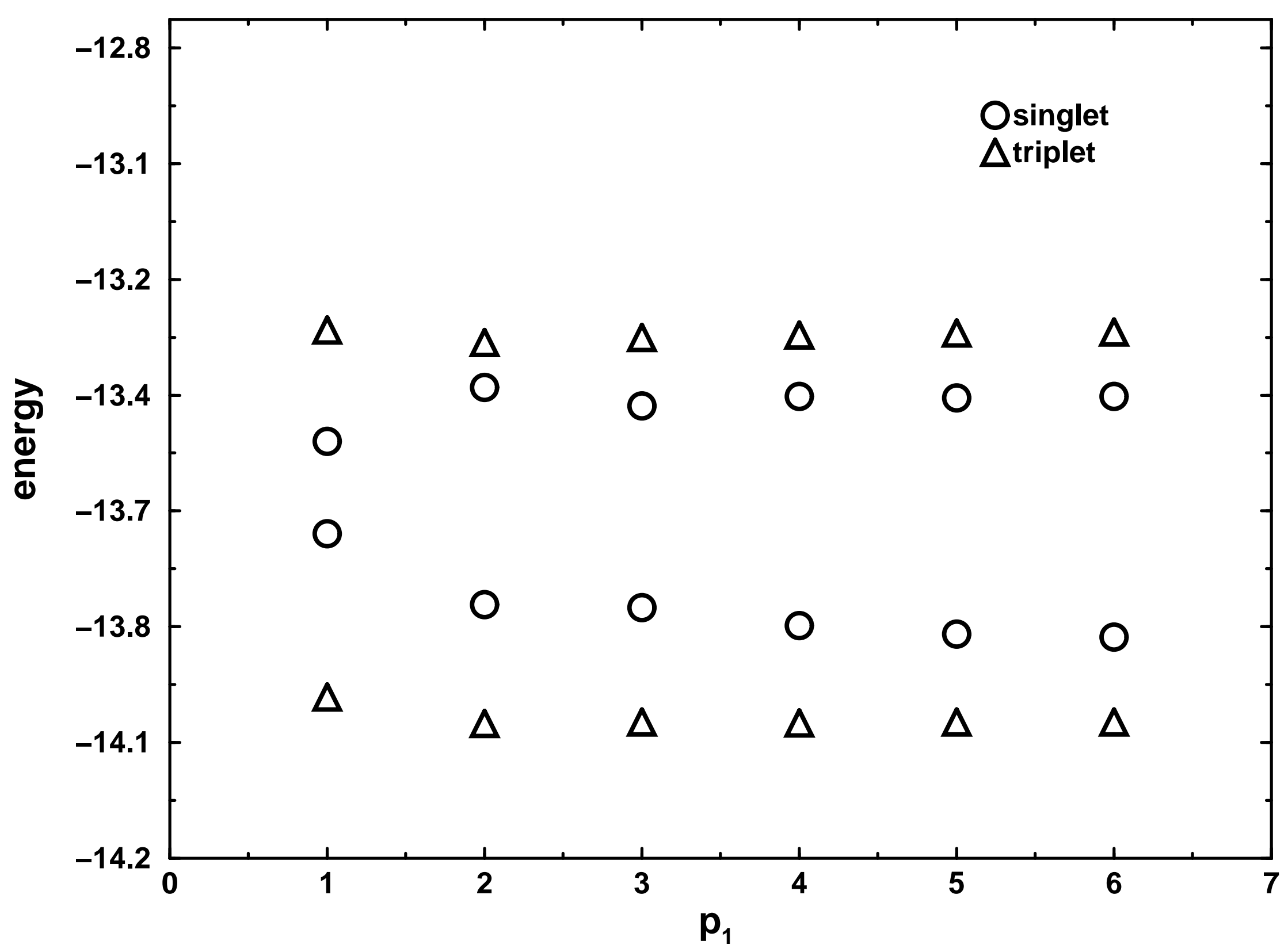


Fig. 3.c

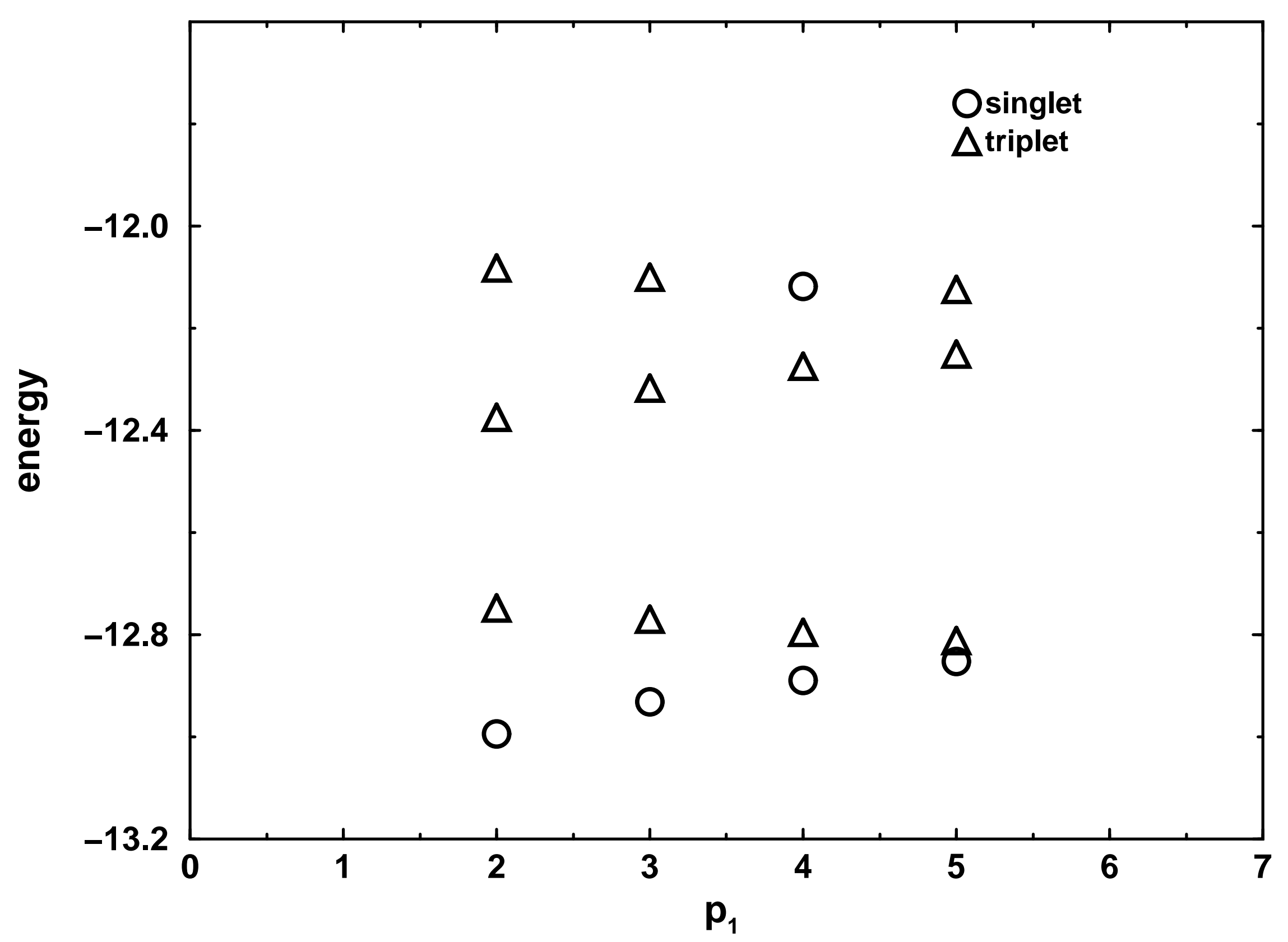


Fig. 3.d

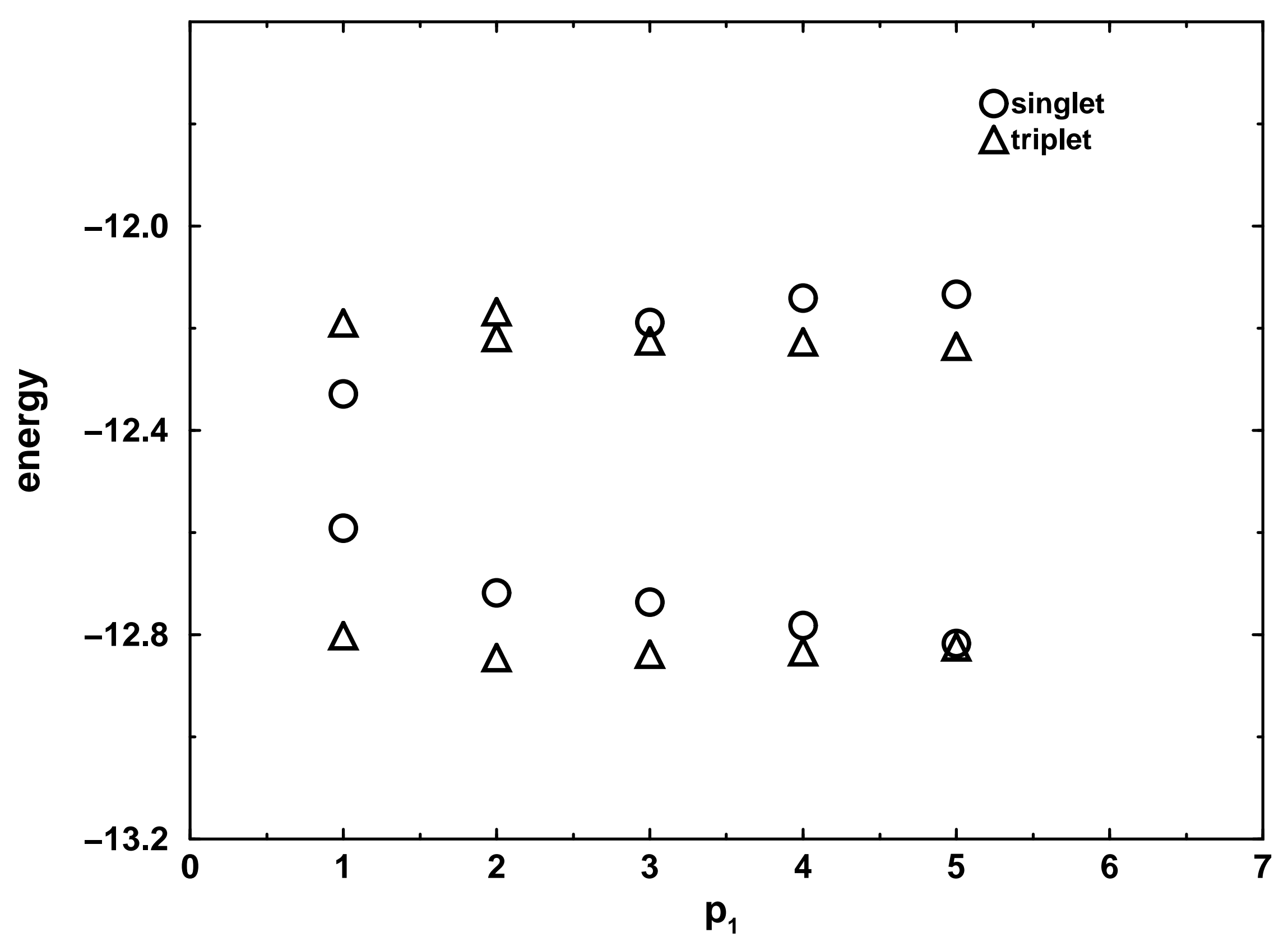


Table 3

\begin{tabular}{|c|c|c|c|c|c|}
\hline \multicolumn{3}{|c|}{$\mathrm{A}(4) \mathrm{F}(3) \mathrm{A}(5)$} & \multicolumn{3}{|c|}{$\mathrm{A}(4) \mathrm{A}(3) \mathrm{A}(5)$} \\
\hline$S^{\text {tot }}$ & ladder & eff. model & $S^{\text {tot }}$ & ladder & eff. model \\
\hline 0 & 0.000 & 0.000 & 0 & 0.000 & 0.000 \\
\hline 1 & 0.079 & 0.07776 & 1 & 0.047 & 0.04730 \\
\hline 1 & 0.131 & 0.12681 & 1 & 0.197 & 0.19146 \\
\hline 2 & 0.198 & 0.19196 & 0 & 0.204 & 0.20080 \\
\hline 1 & & 0.30932 & 1 & 0.269 & 0.26093 \\
\hline 0 & & 0.32193 & 2 & 0.310 & 0.29890 \\
\hline \multicolumn{3}{|c|}{$\mathrm{F}(4) \mathrm{A}(3) \mathrm{A}(5)$} & \multicolumn{3}{|c|}{$\mathrm{F}(4) \mathrm{F}(3) \mathrm{A}(5)$} \\
\hline 1 & 0.000 & 0.000 & 1 & 0.000 & 0.000 \\
\hline 0 & 0.030 & 0.02582 & 2 & 0.075 & 0.07296 \\
\hline 1 & 0.156 & 0.15188 & 0 & 0.094 & 0.09089 \\
\hline 2 & 0.208 & 0.20117 & 1 & 0.134 & 0.12860 \\
\hline 0 & & 0.22053 & 0 & & 0.23404 \\
\hline 1 & 0.302 & 0.29564 & 1 & & 0.26930 \\
\hline \multicolumn{3}{|c|}{$\mathrm{F}(4) \mathrm{F}(3) \mathrm{F}(5)$} & \multicolumn{3}{|c|}{$\mathrm{F}(5) \mathrm{A}(4) \mathrm{F}(5)$} \\
\hline 2 & 0.000 & 0.000 & 0 & 0.000 & $\overline{c 0.000}$ \\
\hline 1 & 0.036 & 0.03474 & 1 & 0.010 & 0.010 \\
\hline 0 & 0.094 & 0.08660 & 2 & 0.109 & 0.107 \\
\hline 1 & 0.108 & 0.10041 & 1 & 0.168 & 0.164 \\
\hline 1 & & 0.22085 & 1 & 0.201 & 0.194 \\
\hline 0 & & 0.26940 & 0 & 0.273 & 0.261 \\
\hline \multicolumn{3}{|c|}{$\mathrm{F}(3) \mathrm{A}(4) \mathrm{F}(3)$} & \multicolumn{3}{|c|}{$\mathrm{A}(5) \mathrm{F}(4) \mathrm{A}(6) \mathrm{F}(4) \mathrm{A}(5$} \\
\hline 0 & 0.000 & $\overline{0.000}$ & 0 & $\overline{0.000}$ & 0.000 \\
\hline 1 & 0.017 & 0.017 & 1 & 0.051 & 0.049 \\
\hline 2 & 0.109 & 0.104 & 1 & 0.088 & 0.081 \\
\hline 1 & 0.225 & 0.216 & 1 & 0.095 & 0.091 \\
\hline 1 & 0.271 & 0.257 & 2 & 0.128 & 0.126 \\
\hline \multirow[t]{14}{*}{0} & 0.403 & 0.387 & 2 & 0.148 & 0.133 \\
\hline & & & 0 & & 0.161 \\
\hline & & & 1 & & 0.166 \\
\hline & & & 2 & 0.196 & 0.182 \\
\hline & & & 3 & 0.216 & 0.202 \\
\hline & & & 1 & & 0.219 \\
\hline & & & 1 & & 0.223 \\
\hline & & & 0 & & 0.226 \\
\hline & & & 0 & & 0.235 \\
\hline & & & 2 & 0.298 & 0.281 \\
\hline & & & 1 & & 0.309 \\
\hline & & & 2 & 0.325 & 0.311 \\
\hline & & & 0 & & 0.398 \\
\hline & & & 1 & & 0.401 \\
\hline
\end{tabular}


Fig. 4

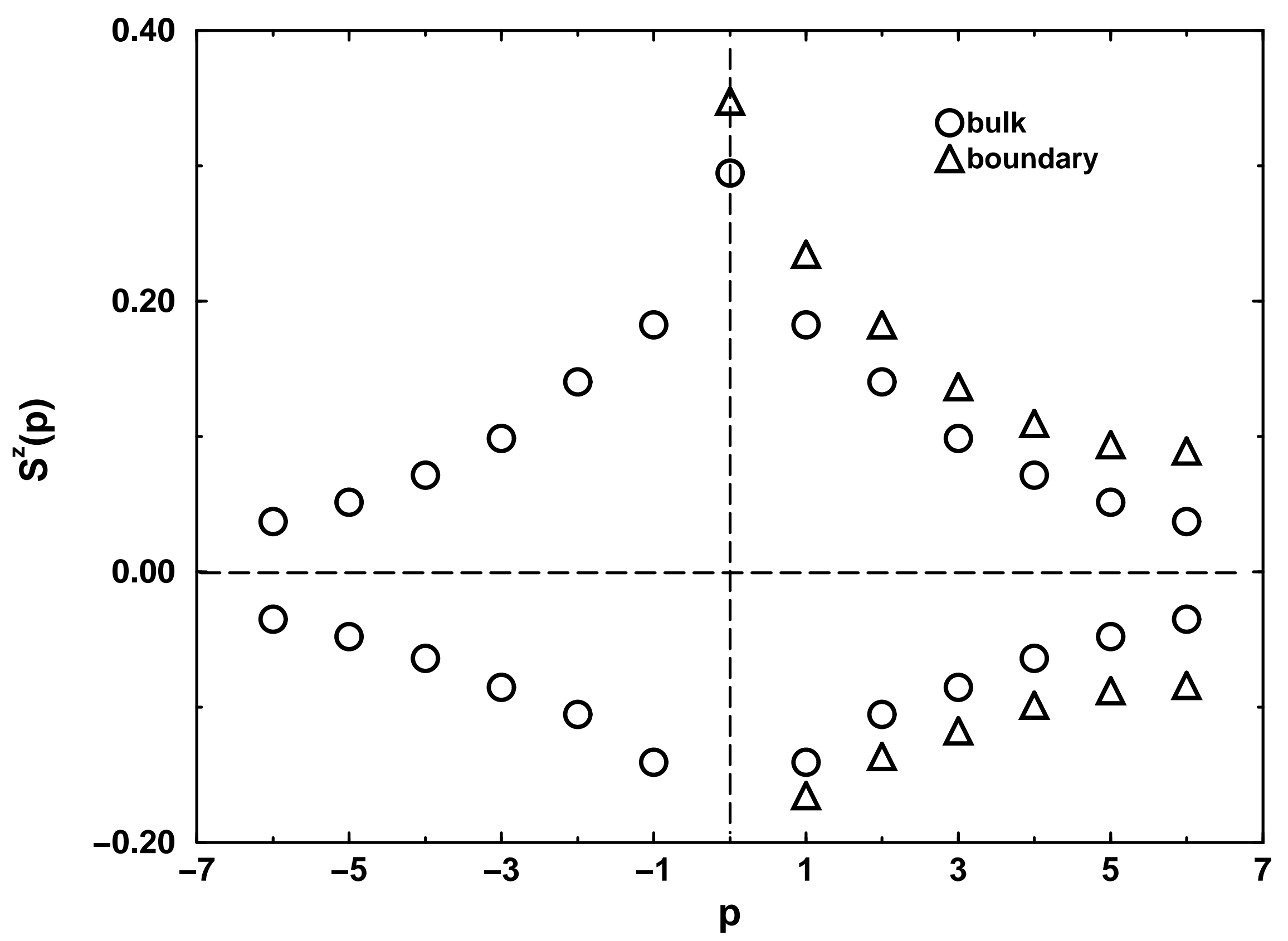


Fig. 5

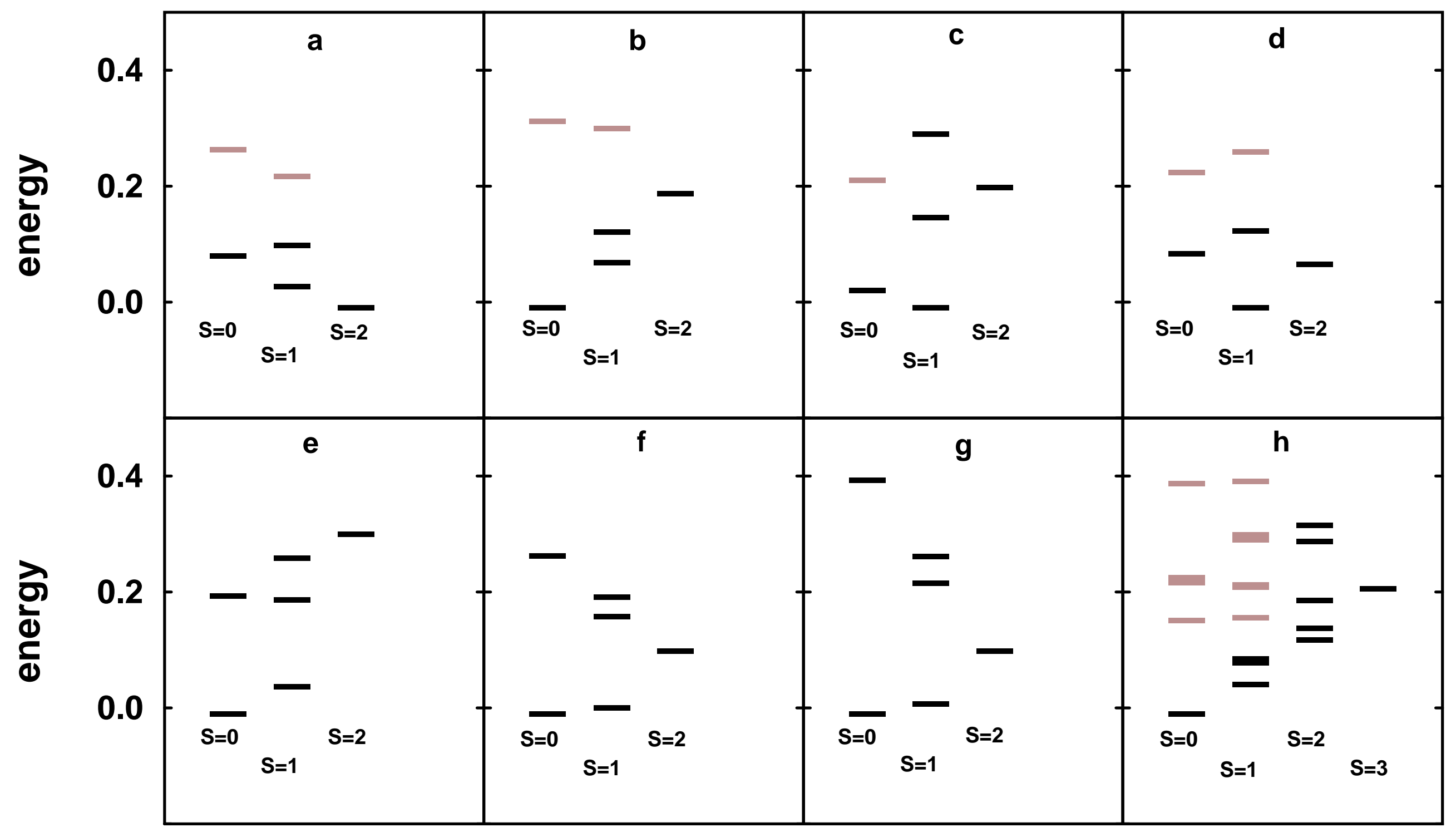


Table 1

\begin{tabular}{|c|c||c|c|}
\hline \multicolumn{2}{|c||}{$S^{z}=0$} & \multicolumn{2}{c|}{$S^{z}=1$} \\
\hline$M=40$ & $M=100$ & $M=40$ & $M=100$ \\
\hline-65.495857 & -65.505486 & -65.089740 & -65.103673 \\
\hline-65.518368 & -65.518391 & -65.118436 & -65.120279 \\
\hline-65.518388 & -65.518391 & -65.120622 & -65.120652 \\
\hline
\end{tabular}

